\title{
122. Free Topological Groups and Infinite Direct Product Topological Groups.
}

\author{
By Shizuo Kakutani. \\ Mathematical Institute, Osaka Impcrial University. \\ (Comm. by T. TaKagi, M.I.A., Oct. 12, 1944.)
}

1. The notion of a free topological group was introduced by A. Markoff ${ }^{11}$, and the existence of a free topological group for any completely regular topological space was established by him. Recently, further investigations were given to this problem by T. Nakayama ${ }^{2}$, who among other things proved that every free topological group is muximally almost periodic. The purpose of this note is to give a simple and direct proof to the existence theorem of A. Markoff. Our method of proof is based on the use of an infinite direct product topological group, and will make it possible to obtain many other known and new results concerning free topological groups almost immediately.

2. A topological space $R$ is a subspace of another topological space $S$, if $R$ is a subset of $S$ and if the relative topology induced on $R$ by that of $S$ is equivalent with the original topology of $R$. It was proved by $\mathrm{E}$. ( Cech' ${ }^{3)}$ that, for any completely regular topological space $R$, there exists a compact Hausdorff space $\bar{R}$ with the following properties:

(1) $R$ is a subspace of $\bar{R}$,

(2) $R$ is dense in $\bar{R}$,

(3) for any continuous mapping $\varphi(x)$ of $R$ into any compact Hausdorff space $S$, there exists a continuous mapping $\Phi(x)$ of $\bar{R}$ into $S$ such that $\Phi(x)=\varphi(x)$ on $R$.

Such a compact Hausdorff space $\bar{R}$ is uniquely determined up to a homeomorphism which leaves every element of $R$ invariant. $\bar{R}$ is called the Cech compactification of $R$.

A topolological group $T$ is toroidal if it is topologically isomorphic with the infinite direct product topological group $\boldsymbol{P}_{r \in \Gamma} K_{r}$, where each $K_{r}$ is a topological group topologically isomorphic with the additive group $K$ of all real numbers mod 1 with the usual compact topology.

Lemma 1. Every completely regular topological space is a subspace of a toroidal group.

Proof. Let $R$ be a completely regular topological space. Let $\left\{\varphi_{r} \mid \gamma \in \Gamma\right\}$ be the family of all continuous mappings $\varphi_{r}(x)$ of $R$ into $K$. Consider the toroidal group $T=\boldsymbol{P}_{r \in \Gamma} K_{r}$, where $K_{r}=K$ for each

1) A. Markoff, On free topological groups, C. R. URSS, 31 (1941), 299-301.

2) T. Nakayama, Note on free topological groups, Proc., 19 (1943), 471-475.

3) E. Čech, On bicompact spaces, Annals of Math., 38 (1937).

S. Kakutani, Concrete representation of abstract $(M)$-spaces and the characterization of the space of continuous functions, Annals of Math., 42 (1941). 
$\gamma \in \Gamma$, and observe a mapping $\varphi(x)$ of $R$ into $T$ defined by $\varphi(x)=$ $\left\{\varphi_{r}(x) \mid \gamma \in \Gamma\right\}$. (This means that the $r$-th coordinate of $\varphi(x) \in T$ is $\left.\varphi_{r}(x) \in K_{r}\right)$. Then it is easy to see that $\varphi(x)$ gives a homeomorphic mapping of $R$ onto $\varphi(R) \subset T$.

Lemma 2. Every completely regular topological space $R$ is a subspace of a totally bounded topological group $G$ such that $\mathfrak{p}(G) \leqq \max \left(\mathfrak{p}(R), \aleph_{0}\right)$.

Proof. Lemma 2 follows from Lemma 1 immediately. We have only to consider the topological subgroup $G$ of $T$ generated by $\varphi(R)<T$.

3. Theorem 1. For any completely regular topological space $R$, there exists a topological group $F$ with the following properties:

(4) $R$ is a subpace of $F$,

(5) $R$ generates $F$ algebraically,

(6) for any continuous mapping $\varphi(x)$ of $R$ into any topological group $G$, there exists a continuous homomorphism $\varphi(x)$ of $F$ into $G$ such that $\Phi(x)=\varphi(x)$ on $R^{4}$.)

Proof. Let $\left\{G_{\gamma} \mid r \in I\right\}$ be the family of all topological groups $G_{\gamma}$ such that $\mathfrak{p}\left(G_{r}\right) \leqq \mathfrak{m}=\max \left(\mathfrak{p}(R), \aleph_{0}\right)$. For any $r \in \Gamma$, let $\left\{\varphi_{r \delta} \mid \delta \in \Delta_{r}\right\}$ be the family of all continuous mappings $\varphi_{r \delta}(x)$ of $R$ into $G_{\gamma}$. Let us further consider the infinite direct product topological group $G=$ $\boldsymbol{P}_{r \in \Gamma} \boldsymbol{P}_{\delta \in \Delta_{\gamma}} G_{r \delta}$, where $G_{r \delta}=G_{r}$ for any $\delta \in \Delta_{r}, r \in \Gamma$, and observe a mapping $\varphi(x)$ of $R$ into $G$ defined by $\varphi(x)=\left\{\varphi_{r \delta}(x) \mid \delta \in \Delta_{r}, \gamma \in \Gamma\right\}$ (This means that the $r \delta$-th coordinate of $\varphi(x) \in G$ is $\left.\varphi_{r \delta}(x) \in G_{r \delta}\right)$. From Lemma 2 follows easily that $\varphi(x)$ gives a homeomorphic mapping of $R$ onto $\varphi(R) \subset G$.

Let now $F$ be the topological subgroup of $G$ generated by $\varphi(R)$. Then $F$ is a required group. In fact, the conditions (4) and (5) are clearly satisfied because of its construction, and (6) follows immediately from the following two facts: (i) without loss of generality we may assume that $\mathfrak{p}(G) \leqq \mathfrak{m}$ in (6), (ii) every mapping $\varphi_{r \delta}(x)$ of $R$ into $G_{r \delta}=G_{r}$ is obtained by taking the $r \delta$-th coordinate of $\varphi(x) \in G$.

It is easy to see that the group $F$ in Theorem 1 is uniquely determined up to a topological isomorphism which leaves every element of $R$ invariant. This group $F$ is called the free topological group of $R$ and is denoted by $F(R)$. From our construction we can see easily that $R$ constitutes a free generating system in $F(R)$ and that $R$ is closed in $F(R)$.

4. By the same argument as in $\$ 3$ we may prove

Theorem 2. Let $R$ be a completely regular topological space. Let further $\mathbb{B}=\{G\}$ be a family of topological groups $G$ with the following properties :

(7) $\mathfrak{p}(G) \leqq \mathfrak{m}=\max \left(\mathfrak{p}(R), \aleph_{0}\right)$ for any $G \in \mathbb{B}$,

(8) if $G$ is a topological subgroup of $\boldsymbol{P}_{\gamma \in \Gamma} G_{r}$, where $G_{\gamma} \in(\mathbb{B}$ for any $r \in \Gamma$ (we do not assume that $G_{r} \neq G_{\gamma^{\prime}}$ for $r \neq \gamma^{\prime}$ ) such that $\mathfrak{H}(G) \leqq \mathfrak{u}$, then $G \in \mathbb{B}$,

4) A. Markoff, loc. cit., 1), Theorem 1. 
(9) there exists a $G \in \mathbb{B}$ which contains $R$ as a subspace.

Then there exists a topological group $F \in \mathbb{B}$ with the properties (4), (5) and

(6') for any continuous mapping $\varphi(x)$ of $R$ into any topological group $G \in(\$)$, there exists a continuous homomorphism $\Phi(x)$ of $F$ into $G$ such that $\Phi(x)=\varphi(x)$ on $R$.

Such a group $F$ is uniquely determined up to a topological isomorphism which leaves every element of $R$ invariant. This group $F$ is called the free (S-group of $R$ and is denoted by $F(R, \mathbb{S})$.

Example 1. Let $\mathbb{S}_{1}$ be the family of all commutative topological groups $G$ such that $\mathfrak{p}(G) \leqq \mathfrak{m}$. Then $F\left(R, \mathbb{S}_{1}\right)$ is the free commutative topological group of $R$.

Example 2. Let $\mathbb{S}_{2}$ be the family of all linear topological spaces $G$ such that $\mathfrak{p}(G) \leqq \mathfrak{m}$. Then $F\left(R, \mathbb{G}_{2}\right)$ is the free linear topological space of $R$.

Example 3. Let $\mathbb{S}_{3}$ be the family of all totally bounded topolngical groups $G$ such that $\mathfrak{p}(G) \leqq \mathfrak{m}$. Then $F\left(R, \mathbb{S}_{3}\right)$ is the free totally bounded topological group of $R$.

Example 4. Let $\mathbb{S}_{4}$ be the family of all topological groups $G$ such that $\mathfrak{p}(G) \leqq \mathfrak{m}$ and $G^{(k)}=(e)$, where $G^{(k)}$ is the $k$-th commutater group of $G$ and $(e)$ is a trivial subgroup of $G$ consisting of the unit element alone. Then $F\left(R, \mathbb{S}_{4}\right)$ is the free $k$-soluble group of $R$.

5. Theorem 3. The free topological group of a completely regular topological space is maximally almost periodic ${ }^{5)}$.

Proof. Let $R$ be a completely regular topological space. It suffices to show that $F\left(R, \mathbb{S}_{3}\right)$ is a continuous isomorphic image of $F(R)$. Let $\Phi(x)$ be a continuous homomorphism of $F(R)$ onto $F\left(R, \mathbb{S}_{3}\right)$ which is the extension of the identity mapping of $R \subset F(R)$ onto $R \subset F\left(R, \mathbb{S}_{3}\right)$. In order to prove that $\Phi(x)$ is an isomorphism, it is sufficient to show that $R$ constitutes a free generating system in $F\left(R, \mathscr{S}_{3}\right)$, and this follows from the fact that for any finite system $\left\{x_{1}, \ldots, x_{n}\right\}<R$ there exists a continuous mapping $\varphi(x)$ of $R$ into a rotation group $G$ (of sufficiently high dimension) such that the image $\left\{\varphi\left(x_{1}\right), \ldots, \varphi\left(x_{n}\right)\right\} \subset G$ constitutes a free system in $G$.

6. A uniform space $R$ (i. e. a topological space $R$ with a uniform structure) is a subspace in the uniform sense of another uniform space $S$ if $R$ is a subset of $S$ and if the relative uniform structure induced on $R$ by that of $S$ is equivalent with the original uniform structure of $R$.

Every topological group and hence every linear topological space may be considered as a uniform space. In case the group $G$ in question is not commutative we shall always consider the left uniform structure of $G$.

Lemma 3. Every uniform space is a subspace in the uniform sense of a linear topological space, and hence of a commutative topological group.

5) T. Nakayama, loc. cit., 2), Theorem 1. 
Proof. This J emma follows easily from the following two facts: (i) every uniform space is a subspace in the uniform sense of an infinite direct product uniform space of metric spaces ${ }^{6}$, (ii) every metric space is isometric with a subset of a normed linear space ${ }^{7}$.

Theorem 4. For any uniform space $R$ there exists a topological group $F$ with the following properties:

(10) $R$ is a subspace in the uniform sense of $F$,

(11) $R$ generates $F$ algebraically,

(12) for any uniformly continuous mapping $\varphi(x)$ of $R$ into any topological group $G$ there exists a continuous (hence uniformly continuous) homomorphism $\Phi(x)$ of $F$ into $G$ such that $\Phi(x)=$ $\varphi(x)$ on $R$.

This theorem may be proved in the same way as Theorem 1. We have only to use Lemma 3 instead of Lemma 1.

The topological group $F$ obtained in Theorem 4 is uniquely determined up to a topological isomorphism which leaves every element of $R$ invariant. This group is called the free topological group in the uniform sense of $R$. It is not difficult to extend Theorem 4 in the same direction as Theorem 2 generalizes Theorem 1, but we shall not enter into the detail of the argument.

6) A. Weil, Sur les espaces a structures uniformes, Actualités, 551, Paris, 1937.

7) K. Kunugui, Applications des espaces à une infinité de dimensions à la théorie des ensembles. Proc., 11 (1935), 351-353.

S. Kakutani, On quasi-metric spaces, Proc. Phys.-Math. Soc. Japan, 19 (1936), 641658 esp. 643. 